Cinémas

Revue d'études cinématographiques

Journal of Film Studies

Villeneuve, Johanne, Brian Neville et Claude Dionne

(direction). La Mémoire des déchets. Essais sur la culture et la

valeur du passé. Québec : Éditions Nota bene, 1999, 243 p.

\title{
Marie Fraser
}

Volume 10, numéro 2-3, printemps 2000

Cinéma et intermédialité

URI : https://id.erudit.org/iderudit/024824ar

DOI : https://doi.org/10.7202/024824ar

Aller au sommaire du numéro

Éditeur(s)

Cinémas

ISSN

1181-6945 (imprimé)

1705-6500 (numérique)

Découvrir la revue

Citer ce compte rendu

Fraser, M. (2000). Compte rendu de [Villeneuve, Johanne, Brian Neville et Claude Dionne (direction). La Mémoire des déchets. Essais sur la culture et la valeur du passé. Québec : Éditions Nota bene, 1999, 243 p.] Cinémas, 10(2-3),

227-233. https://doi.org/10.7202/024824ar d'utilisation que vous pouvez consulter en ligne. 
VILLENEUVE, Johanne, Brian NEVILLE et Claude DIONNE (direction). La Mémoire des déchets. Essais sur la culture et la valeur du passé. Québec: Éditions Nota bene, $1999,243 \mathrm{p}$.

Regroupant des textes de chercheurs issus de cultures diverses et qui œuvrrent dans différents domaines de pensée - en études littéraires, en anthropologie, en histoire de l'art et en philosophie -, ce livre interroge la mémoire en relation avec la culture et propose une réflexion sur l'identité, l'altérité, l'oubli et la valeur du passé. Les auteurs témoignent de conceptions différentes, mais en arrivent tous à reconnaître les enjeux d'une mutation épistémologique de la valeur du passé dans la modernité. Le titre annonce d'entrée de jeu ce glissement: la question du travail de la mémoire y étant posée dans des termes différents, soit sous l'angle du paradigme du "déchet ».

Ce livre, qui donne suite à un colloque qui s'est tenu en mars 1995, est présenté comme une contribution aux recherches actuelles sur la mémoire après la "crise de l'histoire». Il s'inscrit à l'intérieur de tout un débat sur la valeur du passé et celle de l'objet culturel, amorcé dès la fin du XIX ${ }^{c}$ siècle, notamment avec Nietzsche; un débat qui vient aujourd'hui hanter le discours de l'histoire'. Malgré cette hantise, les auteurs ne cherchent pas à jouer la mémoire contre l'histoire, pas plus que la mémoire n'est la relève du discours historique. Le paradigme du déchet engage plutôt à penser les déplacements de l'histoire et de la culture en fonction de leurs restes et de leurs résidus. Ni historicisme ni positivisme, la mémoire s'apparenterait au dispositif du palimpseste, s'activant autant en fonction de l'oubli, du déni que de la remémoration (p. 15). L'ouvrage aborde cette particularité de la mémoire, moderne et postmoderne, en fonction de l'oubli, mais 
aussi en fonction de sa propre intensification prise dans l'utopie de la technologie, voire de la mondialisation et de la globalisation. Dans le contexte d'une fin de millénaire, nous nous retrouvons dans une "impasse épistémique»: l'histoire est aux prises, d'un côté, avec une hétérogénéité et une pluralité des microhistoires et, de l'autre, avec une technologie de plus en plus sophistiquée qui en permet la conservation et la documentation, mais qui gère notre mémoire dans un tout autre espace-temps, qui nous échappe (p. 14). La mémoire serait placée aujourd'hui devant ces deux processus, entre une mémoire publique, collective, et une mémoire individuelle, intime.

Le livre s'ouvre ainsi sur un paradoxe. Depuis la crise de l'histoire, la mémoire se doit de gérer autant la perte et l'oubli qu'une "accumulation en trop", une surdétermination. La question qui émerge alors est de savoir comment gérer à la fois la pluralité et la perte, et quelle valeur leur attribuent la culture et l'histoire. C'est là un des enjeux de la modernité que les auteurs abordent tous d'une manière ou d'une autre. Cette question apparaît d'autant plus pertinente, ou plutôt urgente, parce que, devant la violence des événements qui ont marqué ce siècle (les deux guerres mondiales, les génocides, la Shoah, pour ne nommer que ceux-là), elle atteste d'une exigence mémoriale. La mémoire se trouve donc posée, à travers les essais, en rapport avec un principe de responsabilité, et sur cet horizon se dessinerait un paradigme éthique postmoderne: un "rappel à la mémoire» est toujours, selon l'expression de Jacques Derrida, un «appel à la responsabilité " (p. 21). Le paradoxe d'une mémoire excessive chargée d'oubli et ce paradigme éthique traversent l'ensemble des essais.

La première partie, "L'efficace de la mémoire", traite de la mutation, d'un point de vue historique et épistémologique, de la memoria comme enjeu de la modernité. Si la communauté (et la culture) dans son sens traditionnel repose sur la memoria, la «[...] perte de la mémoire équivaut à une véritable catastrophe», "[...] elle est le signe de sa mort" (p. 27). Lamnésie est en effet terrifiante pour la communauté et pour la tradition. L'activité de la mémoire dans la modernité, moins caractérisée par la collectivité que par l'individu, tend à l'inverse à se réapproprier cette perte; la mémoire se retrouve autant travaillée par 
l'oubli. Avec cette notion de perte qui n'agit plus comme pierre d'achoppement de l'histoire, la mémoire se déplace et se transforme. Quel serait alors son lieu? Quels seraient sa valeur et son efficace? Wlad Godiztch interroge cette efficacité de la mémoire, alors qu'aujourd'hui, il y a perte de son contenu et de sa substance au profit d'une matérialité indépendante de toute forme (p. 37 et 39). Ce texte, qui est le premier de l'ouvrage, (se) joue habilement de cette mutation de la mémoire, ses marques d'oralité allant même à rebours de son contenu, de manière à ce que les choses, par une certaine ironie, se retournent sur elles-mêmes. Ce retournement a pour effet, par contre, de donner plus de force à la mémoire, comme si celle-ci redoublait d'efficacité. La mémoire, aussi hétérogène soit-elle, se présente comme une "machine à voyager dans le temps", selon l'expression de Jacques Derrida (p. 33). De tels déplacements de la mémoire, marquée désormais par une hétérogénéité et par une pluralité, entraînent également des mutations quant au statut de l'événement et de l'événementialité. Éric Méchoulan propose de saisir le rôle du retour de la mémoire dans la pensée moderne à partir de Bergson, Nietzsche et Freud, en fonction de ce nouveau statut de l'événement sur lequel bute l'Histoire. Si la modernité marque un retour de la mémoire, encore faut-il le penser à la suite de Benjamin, d'Adorno et de Derrida, non pas en termes de passé mais de présent et dans la dimension d'avenir que porte cette mémoire, nous ouvrant à l'autre et nous introduisant "[...] à la temporalité spécifique de la promesse" (p. 58). Éric Méchoulan confere à l'événement cette même figure de l'altérité. Marielle Pandolfi pose la question du lieu de la mémoire (au sens anglais de locality ou location, tel qu'il est compris dans les théories critiques contemporaines postcoloniales) et celle de la relation entre corps et mémoire. D'un point de vue anthropologique, et dans le contexte d'une culture du fragment qui s'oppose à une culture de la totalité, mémoire et corps sont nomades. Définissant un espace-temps qui n'est plus normatif ou répressif, ils permettent, selon l'auteure, de redéfinir depuis la modernité, les rapports à l'histoire et à l'oubli.

La deuxième partie examine la valeur de la culture et la valeur du passé à la fin du $\mathrm{XX}^{\mathrm{e}}$ siècle en regard de la notion de recy- 
clage. Toutes les "fins de l'histoire" ne conduiraient-elles pas à l'«idée d'un épuisement culturel» (p. 86) où la notion de recyclage trouverait en bout de ligne sa pertinence? Chacun des essais renvoie ici à une notion ou à un paradigme particulier, soit le déchet comme esthétique, l'authenticité, la territorialité, l'identité, ou le rituel, et tous répondent de la valeur du recyclage. Walter Moser analyse différentes relations d'interaction entre l'art et les déchets dans le domaine de la littérature et des arts visuels. Ce qu'il nomme les "esthétiques des déchets" lui permet de poser autrement la définition de l'œuvre d'art, désamorçant son statut ontologique et la quête du nouveau tels que les ont prônés le projet moderniste et les avant-gardes. Le déchet marque plutôt une forme d'impureté et ouvre sur la temporalité et la matérialité du résidu et du reste. Hans Ulrich Gumbrecht confronte cette "esthétique du déchet " et la notion de recyclage au discours de l'authenticité. Dans cette rencontre, ironiquement, l'authenticité ressort comme un "simulacre de recyclage». La pratique du recyclage n'entraîne pas que la récupération d'objets, elle permet de conserver et de préserver une mémoire, voire une identité. Joseph Paré questionne ces reterritorialisations culturelles en proposant de penser, dans une perspective postcoloniale, les opérations de recyclage en relation étroite avec la notion de trace, comme une "modalité d'actualisation de la reprise" (p. 117) et comme une façon de revoir la problématique identitaire. Le recyclage, comme le développe également Louis Millogo à travers le rituel du "frappeur de dépotoir", permet de faire émerger une certaine profondeur historique. Il apparaît comme un double processus de transformation, agricole et cognitif, qui s'inscrit à même le temps et fait passer le souvenir à la mémoire collective. À partir des liens de cette notion avec le déchet, le reste ou le résidu, les auteurs nous amènent ici à réfléchir sur la matérialité de la mémoire. Cette matérialité et le recyclage d'objets, de formes ou de valeurs du passé seraient ce qui permettrait aujourd'hui la transmission de la mémoire.

La troisième et dernière partie, "Les déchets monumentaux de l'histoire", aborde le problème de la transformation du passé en lien avec le monument en analysant des cas de destruction ou de transformation radicale de sites et d'architectures, d'efface- 
ment des traces du passé et de renversement des utopies. Lespace-temps de la mémoire est encore au cœur de ces préoccupations sur la disparition, l'effacement et l'oubli, mais il donne lieu ici à une remise en question du rôle du monument en tant que mémoire publique, collective, voire institutionnelle. Les auteurs se positionnent tous sur la limite de cette transformation du travail de la mémoire et de la valeur du passé, et soulèvent des enjeux idéologiques et politiques. Karlheinz Barck aborde l'épineuse question de la relation entre le passé et le présent, vue de l'Allemagne. À partir de Heiner Müller et de Walter Benjamin, elle développe, sur des lieux de mémoire autres, une "contre-mémoire" en quelque sorte, toujours en mouvance, et affirme la nécessité d'ouvrir et de repenser les «modes de présentation de l'histoire" (p. 169) en fonction d'une imprévisibilité du passé en regard du temps présent. Elias Khouri pose ce problème de la préservation de la mémoire en lien avec la destruction de Beyrouth, qui a plongé la ville dans une «amnésie architecturale». Après la disparition de monuments publics et l' «[...] effacement des repères de la cité, le seul espace de mémoire qui demeure est celui de la littérature» (p. 153), une littérature qui cherche en réalité à témoigner non pas du passé, mais de l'effacement et de la disparition de ce passé dans le présent.

La question n'est pas seulement de savoir comment négocier avec ce passé ou d'en (re)connaître la valeur, mais comment négocier avec sa disparition. L'effacement des traces du passé amène Magdalena Zaborowska à reconsidérer la fonction du monument public dans une optique post-totalitaire à la lumière des événements entourant l'unification de l'Est et de l'Ouest, symbolisée par la chute du mur de Berlin. Ces événements forcent à revoir la fonction de certains monuments publics, érigés à l'image du despotisme, comme représentation de l'idéologie, du pouvoir, de la violence du stalinisme (p. 217). Ce regard critique jeté sur les monuments de l'histoire motive également la réflexion de Mikhail Ryklin sur la muséification du socialisme et sur le déboulonnage des statues dans l'ancien bloc soviétique. Nous assistons à un renversement des idéologies en une forme d'iconoclasme qui cherche à dissimuler et à effacer les monuments d'un passé sur lesquels ce même peuple s'est auparavant 
érigé. Cette "manipulation des monuments" est aussi le signe d'une "manipulation de l'Histoire» (p. 171). Et devant cet iconoclasme, qui dénie jusqu'à ses propres représentations, les monuments se trouvent paradoxalement muséifiés et transformés en ouvres d'art. Comme le montre d'ailleurs Régine Robin, qui discute des travaux de Jochen Gerz, interrogeant justement la valeur de la mémoire par le truchement d'une remise en cause de la notion traditionnelle de monument, nous assistons à une forme de recyclage de valeurs idéologiques en valeurs culturelles et historiques. Les œuvres publiques de cet artiste déplacent, en effet, le sens du monument en jouant sur la présence autant que l'absence, sur la mémoire autant que la disparition, sollicitant un type de "mémoire active" qui engage chaque individu dans le travail du deuil et de l'oubli. Dans cette dénégation du passé et cette remise en question de la mémoire publique, se pose également la question de l'utopie. Johanne Villeneuve établit ici un parallèle avec le dépotoir. Elle montre, à partir de la figure du "mutant" du film Underground d'Emir Kusturica et des chambres-installations d'Ilya Kabakov, que l'utopie est en réalité non-lieu, d'abord parce qu'elle produit son anti-utopie et qu'elle est la "projection de sa propre dissolution", ensuite parce qu'elle produit des restes (p. 229). Le dépotoir serait aussi un non-lieu qui révèle, peut-être malgré lui, «[...] que quelque chose reste et que ce reste arrive encore à produire quelque chose". La " [...] culture adhère donc simultanément à deux tendances opposées: la production de déchets et la production de la valeur" (p. 242). L'ouvrage se termine sur ces « restes", à la fois déchets et valeurs, et démontre que, dans la culture et l'histoire, subsistent des résidus irréductibles, impossibles à résorber. Si le discours de l'histoire tend à s'achever dans son lien avec le passé, la mémoire à l'inverse ouvre ce même passé à un espace-temps en devenir.

Ces analyses exposent un passé aux prises avec une surdétermination de la mémoire. L'hyperhistoricité que voyait Nietzsche pour échapper au positivisme et certifier de la nécessité d'une conscience critique du temps devient une hypermuséification qui aurait pour contrepartie de transformer ce passé en son contraire, ou plutôt de le liquider en le vidant de son sens politique et idéologique. Cette faille se trouve ici confrontée aux notions 
de recyclage et de déchet, mais aussi à la transformation du rôle et de la fonction du monument, détourné de la commémoration institutionnelle et monumentale pour prendre à témoin les individus. Si l'oubli n'est plus l'envers de la mémoire, comme le propose ce livre, une telle réflexion sur les "déchets" nous force à penser les relations entre éthique et mémoire. Et c'est sans aucun doute dans cette dernière partie que se pose une telle exigence mémoriale, faisant ressortir concrètement le paradigme éthique de la responsabilité.

Marie Fraser

Université de Montréal

\section{NOTE}

1 On peur donner quelques exemples de ce retour de la mémoire aujourd'hui et de cette remise en question du discours de l'histoire qu'il entraîne. Je renvoie notamment à Jean-Pierre Bacot (direction), Travail de la mémoire 1914-1998. Une nécessité dans un siècle de violence (Paris: Autrement, $\left.\mathrm{n}^{\circ} 54,1999\right)$; à Pierre Nora (direction), Les Lieux de mémoire (Paris: Gallimard, 1986-1993); ainsi qu'aux travaux sur la Shoah dont celui de James Young, The Texture of Memory: Holocaust Memorials and Meaning (New Haven: Yale Universiry Press, 1993). Le livre en mentionne plusieurs et représente, ne serait-ce qu'à ce titre, un ouvrage de référence. 